\title{
关于奇次矩形元恢复导数的强超收敛性的进 一步研究
}

\author{
魏继东 (1) (2), 朱起定 (1)* \\ (1) 湖南师范大学数学与计算机科学学院, 长沙 410081 \\ (2) 衡阳师范学院数学系, 衡阳 421000 \\ *通迅作者, E-mail: qd_zhu@sina.com \\ 收稿日期: 2007-06-26; 接受日期: 2008-07-09 \\ 国家自然科学基金 (批准号: 10671065) 资助项目
}

\begin{abstract}
摘要 通过推广林群等的超收玫结果及 Green 函数估计, 对矩形元利用 SPR 技巧给出 了一种强超收玫方法, 证明了在局部对称点上导数具有 $O\left(h^{k+3}\right)(k \geqslant 3$ 为奇数) 的强超收 敛阶及位移具有 $O\left(h^{k+4}\right)(k \geqslant 4$ 为偶数) 的强超收敛性.
\end{abstract}

\begin{abstract}
关键词有限元 强超收敛 局部对称网格 SPR 算子
$\operatorname{MSC}(2000)$ 主题分类 $65 \mathrm{~N} 30$
\end{abstract}

\section{1 引言}

自从 Zienkiewicz 和 $\mathrm{Zhu}^{[1-3]}$ 提出一类有限元块恢复超收玫算子 (SPR) 以来, 数值分析 家们对各类问题提出了各种恢复超收玫算子, 得到了许多超收玫结果. 比如, 文献 [4] 提出了 Polynomial Preserving Recovery (PPR) 方法, 文献 [5,6] 研究了 SPR 方法并且第一次理论上 证明了这个技巧的超收玫性; 文献 $[7,8]$ 还对两点边值问题及矩形网格下 Poisson 方程证明 了在节点处偶次有限元的 SPR 强超收玫性. 而后, 文献 [9] 对矩形网格偶次有限元得到了在 所有对称点上的 SPR 强超收玫性. 最近, 文献 [10] 利用局部对称技巧和 SPR 技巧对二次三 角形元证明了一致三角形元在局部对称点上导数有 $O\left(h^{4-\epsilon}\right)$ 的超收玫性, 文献 [11] 在一致 矩形网格上对奇次矩形元获得了结点导数的 $O\left(h^{k+2}\right)$ 阶强超收玫结果.

从理性上讲, 矩形元导数的超收玫性最佳阶应当是 $O\left(h^{2 k}\right)$. 前面的结果表明, 对于 $k=$ 1,2 , 这个结果是成立的, 对于 $k=3$, 文献 [12] 从实算中证实有 $O\left(h^{6}\right)$ 阶. 对于一维问题, 早 在 70 年代初, 文献 [13,14] 就得到 $O\left(h^{2 k}\right)$ 阶的超收玫性, 值得强调的是, 工程师袁驱 [15] 从 力学角度获得一个校正格式, 使得导数也有 $O\left(h^{2 k}\right)$ 阶的超收玫性, 这说明一维问题已有完 满的结果.

最近, 文献 [16] 对于矩形元利用积分恒等式及插值处理得到了局部对称点上导数 $O\left(h^{k+3}\right)$ 
的强超逼近性:

$$
\left|\partial u^{I}\left(z_{0}\right)-\partial u^{h}\left(z_{0}\right)\right| \leqslant C h^{k+3}|\ln h|^{2}\|u\|_{k+4, \infty}
$$

$(k \geqslant 3$ 为奇数), 这是二维问题的一个创纪录的超逼近结果.

然而, 在一个小单元片内的局部对称点很少 (只有 25 个), 而且随着 $k$ 的增加保持不变, 运用这些对称点不能构作一个具有 $O\left(h^{k+3}\right)$ 阶超收玫性的恢复导数格式. 本文通过对 Green 函数作出更精细的估计, 改进了文献 [16] 的结果, 得到任意阶导数在局部对称点 $z_{0}$ 处的新 超逼近结果:

当 $k \geqslant 3$ 为奇数, 且 $1 \leqslant i \leqslant k$ 为奇数时, 或当 $k \geqslant 4$ 为偶数, 且 $0 \leqslant i \leqslant k$ 为偶数时, 有

$$
\left|\partial^{i} u^{I}\left(z_{0}\right)-\partial^{i} u^{h}\left(z_{0}\right)\right| \leqslant C h^{k+4-i}|\ln h|^{2}\|u\|_{k+4, \infty} .
$$

运用这足够多的资源, 采用文献 [11] 所用的 SPR 后处理法, 构作了导数恢复算子 $R$, 证 得在网格局部对称点 $z_{0}$ 上导数有 $O\left(h^{k+3}\right)$ ( $k \geqslant 3$ 为奇数) 的强超收敛结果:

$$
\left|\partial u\left(z_{0}\right)-R u^{h}\left(z_{0}\right)\right| \leqslant C h^{k+3}|\ln h|^{2}|u|_{k+4, \infty},
$$

从而证实至少对 $k=1,2,3$ 导数均有 $O\left(h^{2 k}\right)$ 阶的超收玫性.

\section{2 矩形元的投影型插值}

设二阶椭圆问题 $L u=f$ 满足正则条件, 其弱形式为: 设 $u \in H_{0}^{1}(\Omega)$, 满足

$$
\begin{aligned}
& a(u, v)=(f, v), \quad \forall v \in H_{0}^{1}(\Omega), \\
& a(u, v)=\int_{\Omega}\left[\sum_{i, j=1}^{2} \alpha_{i j} \partial_{i} u \partial_{j} v+\alpha_{00} u v\right] d x d y, \\
& (f, v)=\int_{\Omega} f \cdot v d x d y,
\end{aligned}
$$

其中 $\Omega \subset \mathbb{R}^{2}$ 是有界光滑区域, 系数 $\alpha_{i j}$ 满足一致椭圆条件.

假定 $\mathcal{T}^{h}$ 是区域 $\Omega$ 上的拟一致剖分, 在区域内部实现了一致的矩形剖分, 设

$$
e=\left(x_{e}-h_{e}, x_{e}+h_{e}\right) \times\left(y_{e}-k_{e}, y_{e}+k_{e}\right)
$$

为任一矩形单元, $Q_{k}(e)=\operatorname{span}\left\{x^{i} y^{j}: 0 \leqslant i \leqslant k, 0 \leqslant j \leqslant k\right\}$, 称

$$
S^{h}(\Omega)=\left\{v \in C(\bar{\Omega}):\left.v\right|_{e} \in Q_{k}(e), \forall e \in \mathcal{T}^{h}\right\}
$$

为双 $k$ 次有限元空间. 方程 (2.1) 的有限元解 $u^{h} \in S_{0}^{h}=S^{h}(\Omega) \cap H_{0}^{1}(\Omega)$, 满足

$$
a\left(u^{h}, v\right)=(f, v), \quad \forall v \in S_{0}^{h} .
$$

为引入矩形元 $e$ 上的投影型插值, 先讨论一维单元

$$
e_{1}=\left(x_{e}-h_{e}, x_{e}+h_{e}\right)
$$

上的投影型插值, 构作 $L^{2}\left(e_{1}\right)$ 上的规范正交多项式系

$$
L_{0}(x)=\sqrt{\frac{1}{2}} h_{e}^{-\frac{1}{2}}, \ldots, L_{i}(x)=\sigma_{i}\left(\frac{d}{d x}\right)^{i}[A(x)]^{i}, \quad i \geqslant 1,
$$

其中 $\sigma_{i}=\sqrt{\frac{2 i+1}{2}} \frac{1}{i !} h_{i}^{-i-\frac{1}{2}}=O\left(h_{e}^{-i-\frac{1}{2}}\right), A(x)=\frac{1}{2}\left[\left(x-x_{e}\right)^{2}-h_{e}^{2}\right]$, 并令

$$
\omega_{0}(x)=1, \ldots, \omega_{i+1}(x)=h_{e}^{-\frac{1}{2}} \int_{x_{e}-h_{e}}^{x} L_{i}(x) d x, \quad i \geqslant 1,
$$


对 $u \in H^{1}\left(e_{1}\right)$ 定义它的 $k$ 阶投影型插值为

$$
i_{k} u(x)=\sum_{j=0}^{k} \beta_{j} \omega_{j}(x), \quad \forall x \in e_{1},
$$

其中

$$
\left\{\begin{array}{l}
\beta_{0}=u\left(x_{e}-h_{e}\right), \\
\beta_{1}=\sqrt{\frac{1}{2}}\left(u\left(x_{e}+h_{e}\right)-u\left(x_{e}-h_{e}\right)\right), \\
\beta_{j}=h_{e}^{\frac{1}{2}} \alpha_{j-1}, \quad j \geqslant 2 .
\end{array}\right.
$$

考虑矩形元 $e=e_{1} \times e_{2}$ 上的投影型插值的构作, 显然

$$
\left\{l_{i}(x) \bar{l}_{j}(y)\right\}
$$

是 $L^{2}(e)$ 空间的完备规范正交多项式系, 其中 $\bar{l}_{j}(y)$ 是对应区间 $e_{2}=\left(y_{e}-k_{e}, y_{e}+k_{e}\right)$ 上的 正交多项式系, 若 $\partial_{1} \partial_{2} u \in L^{2}(e)$, 则有展开式

$$
\begin{aligned}
& \partial_{1} \partial_{2} u=\sum_{i=0}^{\infty} \sum_{j=0}^{\infty} \alpha_{i j} l_{i}(x) \bar{l}_{j}(y) \\
& \alpha_{i j}=\left(\partial_{1} \partial_{2} u, l_{i} \bar{l}_{j}\right)_{e}=\int_{e} \partial_{1} \partial_{2} u l_{i}(x) \bar{l}_{j}(y) .
\end{aligned}
$$

类似一维情形, 利用 Parseval 恒等式, 可得 [17]

$$
u(x, y)=\sum_{i, j=0}^{\infty} \beta_{i j}(u) \omega_{i}(x) \bar{\omega}_{j}(y),
$$

其中 $\beta_{00}=u\left(x_{e}-h_{e}, y_{e}-k_{e}\right), \beta_{i j}=\left|h_{e} k_{e}\right|^{\frac{1}{2}} \alpha_{i-1, j-1}(u)$.

对于 $k \geqslant 1$, 当 $(x, y) \in e\left(\forall e \in \mathcal{T}^{h}\right)$ 时, 定义 $u$ 的双 $k$ 次投影型插值为

$$
i_{k} u(x, y)=\sum_{i, j=0}^{k} \beta_{i j}(u) \omega_{i}(x) \bar{\omega}_{j}(y) .
$$

由文献 [18] 的积分恒等式技巧, 对 $k$ 次矩形投影型插值 $u^{I}=i_{k} u$, 有如下引理:

引理 2.1 对矩形网格及 $\forall v \in S_{0}^{h}$ 有

$$
\begin{aligned}
& \int_{\Omega} \partial_{i}\left(u-u^{I}\right) \partial_{j} v=O\left(h^{k+3}\right) \sum|u|_{k+3, \infty, e}|v|_{2,1, e}, \quad k \geqslant 3, \quad i, j=1,2 ; \\
& \int_{\Omega} \partial_{i}\left(u-u^{I}\right) \partial_{i} v=\left\{\begin{array}{l}
O\left(h^{k+4}\right) \sum|u|_{k+3, \infty, e}|v|_{3,1, e}, \quad k \geqslant 4, \quad i=1,2 ; \\
O\left(h^{k+3}\right) \sum|u|_{k+3, \infty, e}|v|_{2,1, e}, \quad k \geqslant 3,
\end{array}\right. \\
& \int_{\Omega}\left(u-u^{I}\right) v=\left\{\begin{array}{l}
O\left(h^{k+4}\right) \sum|u|_{k+1, \infty, e}|v|_{3,1, e}, \quad k \geqslant 4, \\
O\left(h^{k+3}\right) \sum|u|_{k+1, \infty, e}|v|_{2,1, e}, \quad k \geqslant 3 .
\end{array}\right.
\end{aligned}
$$

\section{Green 函数及离散 Green 函数的估计}

设准 Green 函数 $G_{z}^{*}$ 及 $\partial_{z} G_{z}^{*}$ 的 Galerkin 解分别为 $G_{z}^{h}, \partial_{z} G_{z}^{h} \in S_{0}^{h}(\Omega)$, 满足

$$
\begin{aligned}
& a\left(G_{z}^{*}, v\right)=\left(\delta_{z}^{h}, v\right), \quad a\left(\partial_{z} G_{z}^{*}, v\right)=\left(\partial_{z} \delta_{z}^{h}, v\right), \quad \forall v \in H_{0}^{1}(\Omega) ; \\
& v(z)=a\left(G_{z}^{h}, v\right), \quad \partial_{z} v(z)=a\left(\partial_{z} G_{z}^{h}, v\right), \quad \forall v \in S_{0}^{h} ;
\end{aligned}
$$


其中 $\delta_{z}^{h}$ 为离散 $\delta$ 函数 (参见文献 [19]), 且

$$
\partial_{z} \delta_{z}^{h}=\lim _{\substack{\Delta z \rightarrow 0 \\ \Delta z \| \ell}} \frac{\delta_{z+\Delta z}^{h}-\delta_{z}^{h}}{|\Delta z|}, \quad \partial_{z} G_{z}^{*}=\lim _{\substack{\Delta z \rightarrow 0 \\ \Delta z \| \ell}} \frac{G_{z+\Delta z}^{*}-G_{z}^{*}}{|\Delta z|} .
$$

我们引进高阶导数 $\partial_{z}^{i} G_{z}^{*}$ 及其 Galerkin 逼近 $\partial_{z}^{i} G_{z}^{h}(i \geqslant 2)$, 满足

$$
\begin{aligned}
& a\left(\partial_{z}^{i} G_{z}^{*}, v\right)=\left(\partial_{z}^{i} \delta_{z}^{h}, v\right), \quad \forall v \in H_{0}^{1}(\Omega), \\
& a\left(\partial_{z}^{i} G_{z}^{h}, v\right)=\partial_{z}^{i} v(z), \quad \forall v \in S^{h},
\end{aligned}
$$

其中

$$
\partial_{z}^{i} \delta_{z}^{h}=\lim _{\substack{\Delta z \rightarrow 00 \\ \Delta z \| \ell}} \frac{\partial_{z}^{i-1} \delta_{z+\Delta z}^{h}-\partial_{z}^{i-1} \delta_{z}^{h}}{|\Delta z|}, \quad \partial_{z}^{i} G_{z}^{*}=\lim _{\substack{\Delta z \rightarrow 0 \\ \Delta z \| \ell}} \frac{\partial_{z}^{i-1} G_{z+\Delta z}^{*}-\partial_{z}^{i-1} G_{z}^{*}}{|\Delta z|},
$$

其中 $\ell$ 为任意指定的方向. 由文献 [19], 我们有如下估计:

引理 3.1 当 $k \geqslant 1$ 时, Green 函数及离散 Green 函数满足

$$
\left\|G_{z}^{*}\right\|_{2,1}+\left\|G_{z}^{h}\right\|_{2,1}^{\prime}+\left\|\partial_{z} G_{z}^{h}\right\|^{2}+\left\|\partial_{z} G_{z}^{*}\right\|^{2} \leqslant C|\ln h| .
$$

引理 3.2 对 $\partial_{z}^{i} \delta_{z}^{h}$, 有

$$
\left|\partial_{z}^{i} \delta_{z}^{h}(x)\right| \leqslant C h^{-2-i} e^{-c h^{-1}|x-z|}, \quad 0 \leqslant i \leqslant k .
$$

证明 当 $i=0,1$ 时, 证明见文献 [19], 当 $k \geqslant 2$ 时, 由于 $\delta_{z}^{h}(x)=\delta_{x}^{h}(z)$, 故可展开成

$$
\delta_{z}^{h}(x)=\sum_{i=1}^{L} \sum_{j=1}^{L} a_{i j} \varphi_{i}(x) \varphi_{j}(z),
$$

其中 $a_{i j}=a_{j i}, \varphi_{j}$ 为对应结点 $z_{j}(j=1, \ldots, L)$ 的 $k$ 次有限元空间的基函数, 且

$$
\left|a_{i j}\right|=\left|\delta_{z i}^{h}\left(z_{j}\right)\right| \leqslant C h^{-2} e^{-c h^{-1}\left|z_{i}-z_{j}\right|} .
$$

又

$$
\partial_{z}^{i} \delta_{z}^{h}(x)=\sum_{i=1}^{L} \sum_{j=1}^{L} a_{i j} \varphi_{i}(x) \partial_{z}^{i} \varphi_{j}(z) .
$$

任给 $x \in \Omega$, 引入指标集 $\sigma_{x}=\left\{i: x \in \operatorname{supp}\left(\varphi_{i}\right)\right\}$, 于是有

$$
\begin{aligned}
\partial_{z}^{i} \delta_{z}^{h}(x) \mid & =\left|\sum_{i \in \sigma_{x}} \sum_{j \in \sigma_{z}} a_{i j} \varphi_{i}(x) \partial_{z}^{i} \varphi_{j}(z)\right| \\
& \leqslant C h^{-i} \max _{i \in \sigma_{x}, j \in \sigma_{z}}\left|a_{i j}\right| \leqslant C h^{-i-2} e^{-C h^{-1}|x-z|} .
\end{aligned}
$$

引理 3.3 存在常数 $C>0$, 使得

$$
\left\|\delta_{z}^{h}\right\|_{0, p}+h\left\|\partial_{z} \delta_{z}^{h}\right\|_{0, p}+h^{i}\left\|\partial_{z}^{i} \delta_{z}^{h}\right\|_{0, p} \leqslant C h^{-2+\frac{2}{p}} .
$$

证明 只需证明最后一项, 由 (3.2) 式,

$$
\begin{aligned}
\left\|\partial_{z}^{i} \delta_{z}^{h}\right\|_{0, p} & =\left(\int_{\Omega}\left|\partial_{z}^{i} \delta_{z}^{h}(x)\right|^{p} d x\right)^{\frac{1}{p}} \\
& \leqslant C\left(\int_{\Omega} h^{(-2-i) p} e^{-c h^{-1} p|x-z|} d x\right)^{\frac{1}{p}} \\
& \leqslant C h^{-2-i+\frac{2}{p}}\left(\int_{0}^{\infty} e^{-C t} t d t\right)^{\frac{1}{p}}\left(\frac{1}{p^{2}}\right)^{\frac{1}{p}} .
\end{aligned}
$$

但后一因子有与 $p$ 无关的界, 证毕. 
引进权函数

$$
\phi(x)=\frac{1}{|x-\bar{x}|^{2}+\theta^{2}},
$$

其中 $\bar{x} \in \Omega$ 为固定点 $\theta=\gamma h, \gamma$ 适当大, 且 $0<\phi(x) \in C^{\infty}(\bar{\Omega})$, 对每个实数 $\alpha \in \mathbb{R}$, 引入带权 范数

$$
\|v\|_{\phi^{\alpha}, \Omega}=\left(\int_{\Omega} \phi^{\alpha}|v|^{2} d x\right)^{\frac{1}{2}}
$$

并引入记号

$$
\begin{aligned}
& \left|\nabla^{m} v\right|^{2}=\sum_{|\beta|=m}\left|D^{\beta} v\right|^{2}, \\
& \|v\|_{m, \phi^{\alpha}, \Omega}=\left(\sum_{i=0}^{m}\left|\nabla^{i} v\right|_{\phi^{\alpha}, \Omega}^{2}\right)^{\frac{1}{2}} .
\end{aligned}
$$

权函数有如下性质 (参见文献 [19])

$$
\begin{aligned}
& \int \phi^{\alpha}(x) d x \leqslant C(\alpha-1)^{-1} \theta^{-2(\alpha-1)}, \quad \alpha>1, \\
& \int \phi(x) d x \leqslant C(\beta)|\ln \theta| .
\end{aligned}
$$

引理 3.4 如 $0<\varepsilon<1$, 则

$$
\left\|\partial_{z}^{i} \delta_{z}^{h}\right\|_{\phi^{-1-\varepsilon}} \leqslant C h^{-i+\varepsilon} .
$$

证明 由 (3.2) 式有

$$
\begin{aligned}
\left\|\partial_{z}^{i} \delta_{z}^{h}\right\|_{\phi^{-1-\varepsilon}}^{2} & =\int \phi^{-1-\varepsilon}\left|\partial_{z}^{i} \delta_{z}^{h}\right|^{2} d x \\
& \leqslant C \int\left(|x-z|^{2}+(\gamma h)^{2}\right)^{1+\varepsilon} h^{-4-2 i} e^{-2 C h^{-1}|x-z|} d x \\
& \leqslant C \int_{0}^{\infty}\left(r^{2}+h^{2} \gamma^{2}\right)^{1+\varepsilon} h^{-4-2 i} e^{-2 C h^{-1} r} r d r
\end{aligned}
$$

令 $u=h^{-1} r$, 则

$$
\left\|\partial_{z}^{i} \delta_{z}^{h}\right\|_{\phi^{-1-\varepsilon}}^{2} \leqslant C \int_{0}^{\infty}\left(u^{2}+\gamma^{2}\right) h^{-2 i+2 \varepsilon} e^{-2 C u} u d u \leqslant C h^{-2 i+2 \varepsilon} .
$$

证毕.

引理 3.5 存在 $h_{0} \in(0,1)$, 使得当 $\varepsilon \in(0,1), h \in\left(0, h_{0}\right)$ 时, 有

$$
\left|\partial_{z}^{i} G_{z}^{*}\right|_{\phi^{1-\varepsilon}}^{2}+\left|\nabla^{2}\left(\phi^{\frac{-1-\varepsilon}{2}} \partial_{z}^{i} G_{z}^{*}\right)\right|_{0}^{2} \leqslant C h^{-2 i+2 \varepsilon},
$$

其中 $\mathrm{C}$ 与 $z$ 无关, 但与 $\varepsilon$ 有关.

证明 设 $r=(1+\varepsilon) /(1-\varepsilon), r^{\prime}=(1+\varepsilon) / 2 \varepsilon$, 于是 $\frac{1}{r}+\frac{1}{r^{\prime}}=1$. 记 $g=\partial_{z}^{i} G_{z}^{*}$, 则有

$$
\begin{aligned}
\|g\|_{\phi^{1-\varepsilon}}^{2} & =\int \phi^{1-\varepsilon} g^{2} d x \leqslant\left(\int \phi^{1+\varepsilon} d x\right)^{\frac{1-\varepsilon}{1+\varepsilon}}|g|_{0, \frac{1+\varepsilon}{\varepsilon}}^{2} \\
& \leqslant C\left(\varepsilon^{-1} \theta^{-2 \varepsilon}\right)^{\frac{1-\varepsilon}{1+\varepsilon}}\|g\|_{0, \frac{1+\varepsilon}{\varepsilon}}^{2} .
\end{aligned}
$$

设 $W \in H_{0}^{1}(\Omega)$ 满足

$$
a(v, W)=\left(v,|g|^{\frac{1}{\varepsilon}} \operatorname{sgn} g\right), \quad \forall v \in H_{0}^{1}(\Omega)
$$


令 $q=\frac{2(1+\varepsilon)}{1-\varepsilon}>2, \frac{1}{p}=\frac{1}{q}+\frac{1}{2}$, 即 $p=1+\varepsilon<2$, 则

$$
\begin{aligned}
\|g\|_{0, \frac{1+\varepsilon}{\varepsilon}}^{\frac{1+\varepsilon}{\varepsilon}} & =|a(g, W)|=\left|\left(\partial_{z}^{i} \delta_{z}^{h}, W\right)\right|=\left|\partial_{z}^{i} P W(z)\right| \\
& \leqslant C h^{1-i}\|P W\|_{2} \leqslant C h^{2-i-\frac{2}{p}}\|P W\|_{2, p} \\
& \leqslant C h^{1-i-\frac{2}{q}}\|W\|_{2, p} \leqslant C h^{1-i-\frac{1-\varepsilon}{1+\varepsilon}}\|g\|_{0, \frac{1}{\varepsilon}}^{\frac{1}{\varepsilon}},
\end{aligned}
$$

即

$$
\|g\|_{0, \frac{1+\varepsilon}{\varepsilon}}^{2} \leqslant C h^{2-2 i-\frac{2(1-\varepsilon)}{1+\varepsilon}} .
$$

将 (3.12) 式代回 (3.11) 式得

$$
\begin{aligned}
|g|_{\phi^{1-\varepsilon}}^{2} & \leqslant C\left(\varepsilon^{-1} \theta^{-2 \varepsilon}\right)^{\frac{1-\varepsilon}{1+\varepsilon}} h^{2-2 i-\frac{2(1-\varepsilon)}{1+\varepsilon}} \\
& =C \varepsilon^{-\frac{1-\varepsilon}{1+\varepsilon}} \gamma^{-\frac{2 \varepsilon(1-\varepsilon)}{1+\varepsilon}} h^{2-2 i-\frac{2 \varepsilon(1-\varepsilon)}{1+\varepsilon}-\frac{2(1-\varepsilon)}{1+\varepsilon}} \\
& \leqslant C \varepsilon^{-1} h^{-2 i+2 \varepsilon} .
\end{aligned}
$$

其次利用先验估计

$$
\begin{aligned}
\left|\nabla^{2}\left(\phi^{\frac{-1-\varepsilon}{2}} \partial_{z}^{i} G_{z}^{*}\right)\right|_{0}^{2} & \leqslant C\left\|L\left(\phi^{\frac{-1-\varepsilon}{2}} g\right)\right\|_{0}^{2} \\
& \leqslant \int \phi^{-1-\varepsilon}|L g|^{2} d x+C\|\nabla g\|_{\phi^{-\varepsilon}}^{2}+C\|g\|_{\phi^{-1-\varepsilon}}^{2},
\end{aligned}
$$

由一致椭圆条件并注意 $L g=\partial_{z}^{i} \delta_{z}^{h}$, 有

$$
\begin{aligned}
& \|\nabla g\|_{\phi^{-\varepsilon}}^{2} \leqslant C\left|a\left(g, \phi^{-\varepsilon} g\right)\right|+C\|g\|_{\phi^{-1-\varepsilon}}^{2}, \\
& \left|a\left(g, \phi^{-\varepsilon} g\right)\right|=\left|\left(\partial_{z}^{i} \delta_{z}^{h}, \phi^{-\varepsilon} g\right)\right| \leqslant \frac{1}{2}\left\|\partial_{z}^{i} \delta_{z}^{h}\right\|_{\phi^{-1-\varepsilon}}^{2}+\frac{1}{2}\|g\|_{\phi^{1-\varepsilon}}^{2} .
\end{aligned}
$$

由 (3.13)-(3.16) 式及 (3.7) 式立即得 (3.10) 式.

推论 1 在引理 3.5 的条件下有

$$
\left\|\partial_{z}^{i} G_{z}^{*}\right\|_{2,1} \leqslant C h^{-i} .
$$

证明 设 $g=\partial_{z}^{i} G_{z}^{*}$, 由引理 3.5 有

$$
\int\left|\nabla^{2} g\right| d x \leqslant\left(\int \phi^{1+\varepsilon} d x\right)^{\frac{1}{2}}\left|\nabla^{2} g\right|_{\phi^{-1-\varepsilon}} \leqslant C h^{-\varepsilon} h^{-i+\varepsilon}=c h^{-i} \text {. }
$$

引理 3.6 设 $k \geqslant 2$, 则存在 $h_{0} \in(0,1)$, 使得当 $\varepsilon \in\left(0, \varepsilon_{0}\right)\left(\varepsilon_{0}=1-\frac{2}{q_{0}}\right), h \in\left(0, h_{0}\right)$ 时有

$$
\left\|g-g^{h}\right\|_{1, \phi^{-1-\varepsilon}} \leqslant C h^{1-i+\varepsilon},
$$

其中 $g=\partial_{z}^{i} G_{z}^{*}, C$ 与 $\mathrm{z}$ 无关但与 $\varepsilon$ 有关.

证明 由文献 [19, 第 3 章引理 6$]$ 有

$$
\left\|g-g^{h}\right\|_{1, \phi^{-1-\varepsilon}}^{2} \leqslant C\left\|\psi-\psi^{I}\right\|_{1, \phi^{1+\varepsilon}}^{2},
$$

其中

$$
\psi=\phi^{-1-\varepsilon}\left(g-g^{h}\right)=\phi^{-1-\varepsilon}\left(g-g^{I}\right)+\phi^{-1-\varepsilon}\left(g^{I}-g^{h}\right) \equiv \psi_{1}+\psi_{2} .
$$

再用引理 3.5 及文献 $[19,(3.44)-(3.46)$ 式] 得

$$
\left\|\psi_{1}-\psi_{1}^{I}\right\|_{1, \phi^{1+\varepsilon}}^{2} \leqslant C h^{2}\left\|\nabla^{2} \psi_{1}\right\|_{\phi^{-1-\varepsilon}}^{\prime 2} \leqslant C h^{2}\left\|\nabla^{2} g\right\|_{\phi^{-1-\varepsilon}}^{2} \leqslant C h^{2-2 i+2 \varepsilon} .
$$

由文献 [19] 的 (3.47) 和 (3.48) 式有

$$
\left\|\psi_{2}-\psi_{2}^{I}\right\|_{1, \phi^{1+\varepsilon}}^{2} \leqslant C h^{2-2 i+2 \varepsilon}+C \gamma^{-2}\left\|g-g^{h}\right\|_{1, \phi^{-1-\varepsilon}}^{2} .
$$


将 (3.20) 和 (3.21) 式代入 (3.19) 式, 适当选 $\gamma$ 并回收得 (3.17) 式.

引理 3.7 设 $k \geqslant 2, q_{0}>2$, 则对任意 $z \in \bar{\Omega}$ 有

$$
\left\|\partial_{z}^{i} G_{z}^{*}-\partial_{z}^{i} G_{z}^{h}\right\|_{1, p} \leqslant C h^{1-i-\frac{2}{p^{\prime}}}
$$

其中 $C$ 与 $z$ 无关, $1 \leqslant p<q_{0}, \frac{1}{p}+\frac{1}{p^{\prime}}=1$.

证明 设 $g=\partial_{z}^{i} G_{z}^{*}, g^{h}=\partial_{z}^{i} G_{z}^{h}$, 作函数 $g$ 的插值 $g^{I}$, 由先验估计及 (3.3) 式,

$$
\left\|g-g^{I}\right\|_{1, p} \leqslant C h\left\|\nabla^{2} g\right\|_{0, p} \leqslant C h\left\|\partial_{z}^{i} \delta_{z}^{h}\right\|_{0, p} \leqslant C h^{1-i-\frac{2}{p^{\prime}}} .
$$

其次由逆估计有

$$
\left\|g^{I}-g^{h}\right\|_{1, p} \leqslant C h^{-\frac{2}{p^{\prime}}}\left\|g^{I}-g^{h}\right\|_{1,1} .
$$

上式对 $p=1$ 也成立, 这是因为 (3.17) 式成立, 从而由三角不等式

$$
\left\|g^{I}-g^{h}\right\|_{1, p} \leqslant C h^{1-i-\frac{2}{p^{\prime}}}+C h^{-\frac{2}{p^{\prime}}}\left\|g-g^{h}\right\|_{1,1},
$$

又由 (3.18) 式有

$$
\left\|g-g^{h}\right\|_{1,1} \leqslant C\left(\int \phi^{1+\varepsilon} d x\right)^{\frac{1}{2}}\left\|g-g^{h}\right\|_{1, \phi^{-1-\varepsilon}} \leqslant C h^{-\varepsilon} h^{1-i+\varepsilon} \leqslant C h^{1-i},
$$

故

$$
\left\|g-g^{h}\right\|_{1, p} \leqslant\left\|g-g^{I}\right\|_{1, p}+\left\|g^{I}-g^{h}\right\|_{1, p} \leqslant C h^{1-i-\frac{2}{p^{\prime}}}
$$

证毕

引理 3.8 在引理 3.7 的条件下, 有

$$
\left\|\partial_{z}^{i} G_{z}^{*}\right\|_{2,1}+\left\|\partial_{z}^{i} G_{z}^{h}\right\|_{2,1}^{\prime} \leqslant C h^{-i} .
$$

证明 只需证第 2 式. 设 $g=\partial_{z}^{i} G_{z}^{*}, g^{h}=\partial_{z}^{i} G_{z}^{h}$, 由引理 3.7 和逆估计得

$$
\begin{aligned}
\left\|g-g^{h}\right\|_{2,1}^{\prime} & \leqslant\left\|g-g^{I}\right\|_{2,1}^{\prime}+\left\|g^{I}-g^{h}\right\|_{2,1}^{\prime} \\
& \leqslant C\|g\|_{2,1}+C h^{-1}\left\|g^{I}-g^{h}\right\|_{1,1} \\
& \leqslant C h^{-i}+C h^{-1} C h^{1-i} \leqslant C h^{-i} .
\end{aligned}
$$

由三角不等式得

$$
\left\|g^{h}\right\|_{2,1}^{\prime} \leqslant\|g\|_{2,1}+\left\|g^{h}\right\|_{2,1}^{\prime} \leqslant C h^{-i}
$$

证毕.

引理 3.9 设 $k \geqslant 2,2 \leqslant i \leqslant k$, 则

$$
\left\|\partial_{z}^{i} G_{z}^{*}\right\|_{0}+\left\|\partial_{z}^{i} G_{z}^{h}\right\|_{0} \leqslant C h^{1-i} .
$$

证明 设 $g=\partial_{z}^{i} G_{z}^{*}, \quad g^{h}=\partial_{z}^{i} G_{z}^{h}$, 作 $W \in H_{0}^{1}(\Omega)$ 满足

$$
a(v, W)=(v, g), \quad \forall v \in H_{0}^{1}(\Omega),
$$

则由逆估计及稳定性估计,

$$
\begin{aligned}
\|g\|_{0}^{2} & =a(g, W)=\partial_{z}^{i} P W(z) \\
& \leqslant C h^{1-i}\|P W\|_{2} \leqslant C h^{1-i}\|W\|_{2} \leqslant C h^{1-i}\|g\|_{0},
\end{aligned}
$$

其中 $P$ 为 $L^{2}$ 投影算子, 所以

$$
\|g\|_{0} \leqslant C h^{1-i}
$$


又由三角不等式及 (3.17) 式,

$$
\begin{aligned}
\left\|g^{h}\right\|_{0} & \leqslant\left\|g^{h}-g\right\|_{0}+\|g\|_{0} \leqslant C h^{2}\|g\|_{2}+\|g\|_{0} \\
& \leqslant C h^{2}\left\|\partial_{z}^{i} \delta_{z}^{h}\right\|_{0}+\|g\|_{0} \leqslant C h^{2} C h^{-1-i}+C h^{1-i} \leqslant C h^{1-i} .
\end{aligned}
$$

\section{4 局部对称点及超逼近性}

设 $D \subset \Omega$ 为剖分网格 $\mathcal{T}^{h}$ 的子域, 我们称网格 $D$ 关于点 $z_{0}$ 为局部对称的, 若对 $v \in$ $S^{h}(D)$ 有

$$
\hat{v} \stackrel{\text { def }}{=} v\left(z_{0}-\left(x-z_{0}\right)\right) \in S^{h}(D) .
$$

文献 $[16]$ 得到如下引理:

引理 4.1 若存在常数 $C_{0}$, 使得 $|x-z| \geqslant C_{0} h$, 则有

$$
\begin{aligned}
& \left|\partial^{3} G_{z}^{h}(x)\right| \leqslant C|\ln h|(x-z)^{-3}, \\
& \left|\partial^{2} \partial_{z} G_{z}^{h}(x)\right| \leqslant C|\ln h|(x-z)^{-3},
\end{aligned}
$$

进一步, 利用引理 3.7-3.9 及文献 [16] 的同样方法, 还有

引理 4.2 在引理 4.1 的条件下, 有

$$
\left|\partial^{2} \partial_{z}^{i} G_{z}^{h}(x)\right| \leqslant C h^{1-i}|\ln h|(x-z)^{-3}, \quad 1 \leqslant i \leqslant k .
$$

\section{证明 见附录.}

为简便起见, 不妨设整个区域 $\Omega$ 上的网格剖分 $\mathcal{T}^{h}$ 都是关于 $z_{0}$ 对称的. 事实上, 对任何 局部对称网格, 可以构造截断函数 $\omega$, 使得在 $z_{0}$ 的邻域为 1 , 支集 $\operatorname{supp}(\omega)$ 为关于 $z_{0}$ 对称网 格的子域, 设 $u_{1}=\omega u$, 则 $u_{1}$ 满足我们的条件.

定理 4.1 设方程 $(2.1)$ 中 $\alpha_{i j}=0(i \neq j), u^{h}$ 和 $u^{I}$ 分别为方程的有限元解和 $k$ 次矩 形投影型插值, $\mathcal{T}^{h}$ 为关于 $z_{0}$ 的矩形对称网格, 则当 $k \geqslant 3$ 是奇数时, 有

$$
\left|\partial^{i} u^{I}\left(z_{0}\right)-\partial^{i} u^{h}\left(z_{0}\right)\right| \leqslant C h^{k+4-i}|\ln h|^{2}\|u\|_{k+4, \infty}, \quad 1 \leqslant i \leqslant k \text { 为奇数. }
$$

当 $k \geqslant 4$ 为偶数时有

$$
\left|\partial^{i} u^{I}\left(z_{0}\right)-\partial^{i} u^{h}\left(z_{0}\right)\right| \leqslant C h^{k+4-i}|\ln h|^{2}\|u\|_{k+4, \infty}, \quad 0 \leqslant i \leqslant k \text { 为偶数. }
$$

证明 当 $k$ 和 $i$ 为奇数时, 设 $v=\partial_{z}^{i} G_{z}^{h}, B=\operatorname{ball}\left(z_{0}, d_{0}\right), d_{0}=c h$, 令

$$
\bar{u}=(u-\hat{u}) / 2, \quad \hat{u}=u\left(z_{0}-\left(x-z_{0}\right)\right),
$$

则 $\bar{u}$ 为奇函数, 对奇数 $m=1,3, \ldots, \partial^{k+m} \bar{u}$ 仍为奇函数, 故

$$
|\bar{u}|_{k+m, \infty, e} \leqslant C \operatorname{dist}\left(z_{0}, e\right)|u|_{k+m+1, \infty, \Omega},
$$

其中 $\operatorname{dist}\left(z_{0}, e\right)$ 表示 $z_{0}$ 和 $e$ 的距离, 利用引理 2.1 和 (4.6) 式有

$$
\begin{aligned}
\left|\partial^{i} \bar{u}^{h}\left(z_{0}\right)-\partial^{i} \bar{u}^{I}\left(z_{0}\right)\right|= & a\left(\bar{u}^{h}-\bar{u}^{I}, v\right)=a\left(\bar{u}-\bar{u}^{I}, v\right) \\
= & O\left(h^{k+3}\right) \sum\left(|\bar{u}|_{k+3, \infty, e}+|\bar{u}|_{k+1, \infty, e}\right)|v|_{2,1, e} \\
= & O\left(h^{k+3}\right)\|u\|_{k+4, \infty, \Omega}\left\{\sum_{e \subset B} C h|v|_{2,1, e}\right. \\
& \left.+\sum_{e \nsubseteq B} \operatorname{dist}\left(z_{0}, e\right)|v|_{2,1, e}\right\} .
\end{aligned}
$$


由引理 3.8 可得

$$
\sum_{e \subset B} C h|v|_{2,1, e} \leqslant C h C h^{-i} \leqslant C h^{1-i} .
$$

由引理 4.2 有

$$
\sum_{e \nsubseteq B} \operatorname{dist}\left(z_{0}, e\right)|v|_{2,1, e} \leqslant C h^{1-i}|\ln h| \int_{\Omega-B} \frac{1}{\left|z_{0}-x\right|^{2}} d x \leqslant C h^{1-i}|\ln h|^{2} .
$$

由于 $\partial^{i} \bar{u}$ 为偶函数, 所以

$$
\partial^{i} u^{h}\left(z_{0}\right)-\partial^{i} u^{I}\left(z_{0}\right)=\partial^{i} \bar{u}^{h}\left(z_{0}\right)-\partial^{i} \bar{u}^{I}\left(z_{0}\right) .
$$

结合 (4.7)-(4.10) 式, (4.4) 式得证. 类似证得 (4.5) 式.

利用同样的方法可得如下定理:

定理 4.2 在定理 4.1 的假设下对 $\alpha_{i j} \neq 0(i \neq j), k \geqslant 3$ 为奇数时有

$$
\left|\partial u^{I}\left(z_{0}\right)-\partial u^{h}\left(z_{0}\right)\right| \leqslant C h^{k+2}|\ln h|^{2}\|u\|_{k+4, \infty} .
$$

\section{5 奇次矩形元导数恢复算子及强超收敛性}

设 $E=\bigcup_{i=1}^{4} e^{(i)}$ 为内结点 $z_{0}=\left(x_{0}, y_{0}\right)$ 处的单元片 (如图 1), 构作线单元片

$$
E_{1}=\left\{(x, y) \in E: y=y_{0}\right\}, \quad E_{2}=\left\{(x, y) \in E: x=z_{0}\right\} .
$$

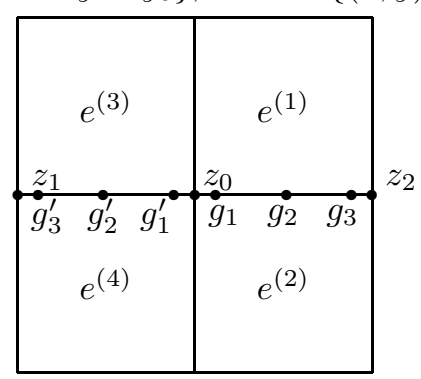

图 1

对奇次矩形元, 分别对线单元片 $E_{1}$ 和 $E_{2}$ 采用样本点集

$$
\begin{aligned}
& G_{1}=\bigcup_{i=1,3}\left\{\left(x, y_{0}\right) \in \bar{e}^{(i)}: \alpha_{k}^{1} l_{k}(x)+\alpha_{k+1}^{1} l_{k+1}(x)=0\right\}, \\
& G_{2}=\bigcup_{i=1,2}\left\{\left(x_{0}, y\right) \in \bar{e}^{(i)}: \alpha_{k}^{2} \bar{l}_{k}(y)+\alpha_{k+1}^{1} \bar{l}_{k+1}(y)=0\right\},
\end{aligned}
$$

其中 $\alpha_{m}^{1}=\int_{e_{1}}\left(x-x_{0}\right)^{k+1} l_{m}(x) d x, \alpha_{m}^{2}=\int_{e_{2}}\left(y-y_{0}\right)^{k+1} \bar{l}_{m}(y) d y(m=k, k+1), e_{1}$ 和 $e_{2}$ 为矩形 元 $e$ 的两条边, 利用 $G_{i}(i=1,2)$ 分别构作 $x$ 方向与 $y$ 方向的导数恢复算子. 任给 $\omega \in S^{h}(E)$, 在 $G_{i}(i=1,2)$ 上构作极小问题

$$
\left\|\left|v_{i}-\partial_{i} \omega \|\right|_{i}=\min \left\{\left\|\left|v-\partial_{i} \omega \|\right|_{i}: v \in P_{k+1}\left(E_{i}\right)\right\},\right.\right.
$$

其中 $\|v\| \|_{i}^{2}=\sum_{g \in G_{i}}[v(g)]^{2}$. 由于 $k>1$ 为奇数, $2 k \geqslant k+3$, 这个最小二乘问题存在唯一解, 故可定义算子

$$
R_{i}: \omega \in S^{h}(E) \longrightarrow v_{i} \in P_{k+1}\left(E_{i}\right)
$$

容易证明如下性质 ${ }^{[10]}$ : 
r1) 若 $v \in P_{k+2}\left(E_{i}\right)$, 则

$$
\left.v\right|_{E_{i}} \equiv 0 \Longleftrightarrow v(g)=0, \forall g \in G_{i} \Longleftrightarrow\|\mid v\|_{i}=0,
$$

且 $R_{i} v=\partial_{i} v$;

r2) 若令 $\langle v, \omega\rangle_{i}=\sum_{g \in G_{i}} v(g) \omega(g)$, 则问题 5.2 等价于方程

$$
\left\langle\partial_{i} \omega-R_{i} \omega, v\right\rangle_{i}=0, \quad \forall v \in P_{k+1}\left(E_{i}\right),
$$

其中 $R_{i} \omega=v_{i}$;

r3) $\left\|\left|R_{i} \omega\left\|\left.\right|_{i} \leqslant\right\| \partial_{i} \omega\|\|_{i} ;\right.\right.$

$\mathrm{r} 4)$ 若 $\omega$ 关于点 $z_{0}$ 偶对称, 则 $R_{i} \omega$ 关于点 $z_{0}$ 奇对称, 从而 $R_{i} \omega\left(z_{0}\right)=0$.

引理 5.1 当 $k>1$ 为奇数时, 算子 $R_{i}$ 满足如下两个条件:

A) $\left|R_{i}\left(u^{I}-u^{h}\right)\left(z_{0}\right)\right| \leqslant C h^{k+3}|\ln h|^{2}\|u\|_{k+4, \infty}$,

B) $\partial_{i} u\left(z_{0}\right)-R_{i} u^{I}\left(z_{0}\right)=0, \forall u \in P_{k+3}\left(E_{i}\right)$,

此处 $u^{I}=i_{k} u$ 是二维插值在线段 $E_{i}$ 上的限制, 事实上是一维 $k$ 阶投影型插值.

证明 不妨设 $i=1, x$ 方向线单元片为 $[-h, h], z_{0}=(0,0)$ 为局部对称点, $\omega=u^{I}-u^{h} \in$ $S^{h}(E)$, 令 $\omega=\left(w(x)+w\left(z_{0}-\left(x-z_{0}\right)\right)\right) / 2+\left(w(x)-w\left(z_{0}-\left(x-z_{0}\right)\right)\right) / 2 \equiv \omega_{1}+\omega_{2}$, 由于 $\omega_{1}$ 为偶函数, 故 $R_{1} \omega_{1}$ 为奇函数, 即 $R_{1} \omega_{1}\left(z_{0}\right)=0$, 故不妨设 $\omega$ 为奇函数. 由于 $\partial_{1} \omega$ 和 $R_{1} \omega$ 为 偶函数, 设

$$
\begin{aligned}
& \partial_{1} \omega=\left\{\begin{array}{l}
b_{0}+b_{1} x+\cdots+b_{k-1} x^{k-1}, \quad x \in(0, h), \\
b_{0}-b_{1} x+\cdots+(-1)^{k-1} b_{k-1} x^{k-1}, \quad x \in(-h, 0],
\end{array}\right. \\
& R_{1} \omega=a_{0}+a_{2} x^{2}+\cdots+a_{k+1} x^{k+1},
\end{aligned}
$$

直接计算可得 $a_{0}=c_{0} b_{0}+c_{1} b_{1} h+\cdots+c_{k-1} b_{k-1} h^{k-1}$, 其中 $c_{i}$ 为常数, 又 $R_{1} \omega(0)=a_{0}, \partial_{1}^{i} \omega(0)=$ $b_{i-1}(1 \leqslant i \leqslant k)$, 由定理 4.1 得

$$
\begin{aligned}
\left|R_{1} \omega(0)\right| & =\left|\sum_{i=0}^{k-1} c_{i} b_{i} h^{i}\right| \leqslant\left.\left|\sum_{i=0}^{k-1} c_{i} C h^{k+4-1-i} h^{i}\right| \ln h\right|^{2}\|u\|_{k+4, \infty} \mid \\
& \leqslant C h^{k+3}|\ln h|^{2}\|u\|_{k+4, \infty} .
\end{aligned}
$$

A) 证毕. 下证 B), 不妨设 $i=1$, 当 $u \in P_{k}\left(E_{1}\right)$ 时, $u^{I}=u$ 成立, 故只需对 $u=u\left(x, y_{0}\right)=$ $\left(x-x_{0}\right)^{m}(m=k+1, k+2, k+3)$ 验证 $\partial_{1} u\left(z_{0}\right)-R_{1} u^{I}\left(z_{0}\right)=0$ 即可. 由于 $k+1, k+3$ 是偶数, 由性质 $\mathrm{r} 4$ ) 得证, 故只需验证 $m=k+2$ 的情形, 由一维投影型插值定义和样本点集 $G_{1}$ 得

$$
\partial_{1} u\left(x, y_{0}\right)=\partial_{1} i_{k} u\left(x, y_{0}\right)+m \alpha_{k}^{1} l_{k}(x)+m \alpha_{k+1}^{1} l_{k+1}(x)=\partial_{1} u^{I}\left(x, y_{0}\right), \quad \forall x \in G_{1},
$$

于是由算子 $R_{1}$ 的定义得 $R_{1} u=R_{1} u^{I}$, 又 $\partial_{1} u \in P_{k+1}\left(E_{1}\right)$, 所以 $\partial_{1} u=R_{1} u=R_{1} u^{I}$. 于是 $\left.\mathrm{B}\right)$ 得证.

综上所述, 我们有如下重要定理:

定理 5.1 设 $k>1$ 为奇数, $u^{h} \in S^{h}(\Omega)$ 是 $u$ 的有限元逼近, 导数恢复算子 $R_{i}$ : $S^{h}\left(E_{i}\right) \rightarrow v_{i} \in P_{k+1}\left(E_{i}\right)(i=1,2)$ 满足条件 A) 和 B), 一致矩形网格 $\mathcal{T}^{h}$ 的子域 $D$ 关于 $z_{0}$ 局部对称, 则当 $E \subset \subset D$ 时, 有超收玫估计

$$
\left|\partial_{i} u\left(z_{0}\right)-R_{i} u^{h}\left(z_{0}\right)\right| \leqslant C h^{k+2}|\ln h|^{2}|u|_{k+4, \infty, \Omega} .
$$

进一步, 若 $\alpha_{i j}=0(i \neq j)$ 时, 有导数的强超收玫性

$$
\left|\partial_{i} u\left(z_{0}\right)-R_{i} u^{h}\left(z_{0}\right)\right| \leqslant c h^{k+3}|\ln h|^{2}|u|_{k+4, \infty, \Omega} .
$$


证明 只需证第 2 个结果就可以了. 由条件 B) 并用 Bramble-Hilbert 引理得

又由条件 A) 得

$$
\left|\partial_{i} u\left(z_{0}\right)-R_{i} u^{I}\left(z_{0}\right)\right| \leqslant C h^{k+3}|u|_{p+4, \infty, \Omega},
$$

$$
\left|R_{i}\left(u^{I}-u^{h}\right)\left(z_{0}\right)\right| \leqslant C h^{k+3}|\ln h|^{2}|u|_{k+4, \infty, \Omega} .
$$

利用三角不等式得证结果.

由于在网格结点 $z_{0}$ 处有 $u\left(z_{0}\right)=u^{I}\left(z_{0}\right)$, 由定理 4.1 及三角不等式有

定理 5.2 设 $k \geqslant 4$ 为偶数, $u^{h} \in S^{h}(\Omega)$ 是 $u$ 的有限元逼近, 一致矩形网格 $\mathcal{T}^{h}$ 的子域 $D$ 关于 $z_{0}$ 局部对称, 则当 $E \subset \subset D, \alpha_{i j}=0(i \neq j)$ 时, 有位移的强超收玫性

$$
\left|u\left(z_{0}\right)-u^{h}\left(z_{0}\right)\right| \leqslant c h^{k+4}|\ln h|^{2}|u|_{k+4, \infty, \Omega} .
$$

\section{6 数例分析}

数例 1 考虑模型问题

$$
\begin{aligned}
& -\Delta u=f, \quad(x, y) \in \Omega=[0,1] \times[0,1], \\
& \left.u\right|_{\partial \Omega}=0 .
\end{aligned}
$$

假设 $f$ 是对应于真解 $u=10 x \sin \pi x \sin \pi y$ 的函数, 设在 $\Omega$ 上实现了一致矩形剖分, 运用双三 次矩形元求解, 考察在点 $x_{0}=(0.25,0.25)$ 的情形 (见表 1 , 参见文献 [20]).

表 1 双三次元在点 $z_{0}=(0.25,0.25)$ 处沿 $x$ 方向的导数误差表

\begin{tabular}{ccc}
\hline$h$ & $\left|\sigma-\sigma^{*}\right|$ & $\left|\sigma-\sigma^{R}\right|$ \\
\hline$\frac{1}{4}$ & $4.137179297103444(-5)$ & $3.875638802971793(-2)$ \\
$\frac{1}{8}$ & $6.474424836255821(-7)$ & $2.481111253091581(-3)$ \\
$\frac{1}{12}$ & $5.575402539648167(-8)$ & $4.922390230728269(-4)$ \\
$\frac{1}{16}$ & $9.788458044113213(-9)$ & $1.559844826211077(-4)$ \\
\hline 收敛速度 & 6 & 4 \\
\hline
\end{tabular}

表 1 中 $\sigma$ 表示真解沿 $x$ 方向的导数, $\sigma^{R}$ 表示用 SPR 产生的导数, $\sigma^{*}$ 为本文方法产生 的恢复导数, 从表 1 可知, 恢复值 $\sigma^{*}$ 有强超收玫性, 收玫阶为 $O\left(h^{6}\right)$.

数例 2 仍然考虑模型问题 (6.1), 将区域一致剖分成 $N_{i} \times N_{i}$ 个正方形, 取双四次矩形 元求解, 考察在点 $z_{0}=(0.25,0.25)$ 处的有限元解 $u^{h}$ 与真解 $u$ 的误差 $E_{N_{i}}$ 的情形 (见表 2 ), 由表 2 可知, 双四次元具有 $O\left(h^{8}\right)$ 的精度.

表 2 双四次矩形元在点 $z_{0}=(0.25,0.25)$ 处有限元解误差表

\begin{tabular}{ccc}
\hline$N_{i}$ & $E_{N_{i}}=\left|u\left(z_{0}\right)-u^{h}\left(z_{0}\right)\right|$ & Rate $=\log _{2}\left(E_{N_{i-1}} / E_{N_{i}}\right)$ \\
\hline$N_{1}=4$ & $1.949919403720912(-8)$ & \\
$N_{2}=8$ & $7.703726545571499(-11)$ & 7.984 \\
$N_{3}=16$ & $3.119726699196690(-13)$ & 7.948 \\
\hline
\end{tabular}

\section{参考文献}

1 Zienkiewicz O C, Zhu J Z. The superconvergence patch recovery and a posteriori estimate. Part 1: The recovery technique. Int J Numer Methods Engrg, 33: 1331-1364 (1992) 
2 Zienkiewicz O C, Zhu J Z. The superconvergence patch recovery and a posteriori estimate. Part 2: Error estimates and adaptivity. Int J Numer Methods Engrg, 33: 1365-1382 (1992)

3 Zienkiewicz O C, Zhu J Z. The superconvergence patch recovery(SPR) and adaptive finite element refinement. Comput Methods Appl Mech Engrg, 101: 207-224 (1992)

4 Zhang Z M, Naga A. A new finite element gradient recovery method: Superconvergence property. SIAM J Sci Comput, 26: 1192-1213 (2005)

5 Zhang Z M, Zhu J Z. Analysis of the superconvergence patch recovery techniques and a posteriori error estimator in the finite element method(I). Comput Methods Appl Mech Engrg, 123: 173-187 (1995)

6 Zhang Z M, Zhu J Z. Analysis of the superconvergence patch recovery techniques and a posteriori error estimator in the finite element method(II). Comput Methods Appl Mech Engrg, 163: 159-170 (1998)

7 Zhang Z M. Ultraconvergenc of the patch recovery technique. Math Comput, 65: 1431-1437 (1996)

8 Zhang Z M. Ultraconvergenc of the patch recovery technique II. Math Comput, 69: 141-158 (2000)

9 Zhang Z M, Lin R. Ultraconvergence of the Zienkiewicz-Zhu patch recovery at symmetry points. Numer Math, 95: 781-801 (2003)

10 Zhu Q D, Meng L X. The derivative ultrarconvergence for quadratic triangular finite elements. J Comput Math, 22: 857-864 (2004)

11 朱起定, 孟令雄, 奇次矩形元导数恢复算子的新构造及其强超收敛性. 中国科学 A 辑: 数学, 34: 723-731 (2004)

12 Meng L X, Zhu Q D. The ultraconvergent derivative recovery technique for odd-older elements: A computerbased test result. Nat Sci J Xiangtan Univ, 25: 117-121 (2003)

13 Douglas J J, Dupont T. Superconvergence for Galerkin methods for the two point boundary problem vie local projietions. Numer Math, 21: 270-278 (1973)

14 Douglas J J, Dupont T. Some superconvergence results for Galerkin methods for the approximate solution of two-point boundary value problems. In: Topics in Numerical Analysis. London: Academic Press, 1973, 89-93

15 袁驷, 王旭, 邢沁妍. 具最佳超收敛阶的 EEP 法格式— I, II, III (2007)(待发表)

16 Lin Q, Zhou J M. Superconvergence in high-order Galerkin finite element methods. Comput Meth Appl Mech Engin, 196: 3779-3784 (2007)

17 林群, 朱起定. 有限元的预处理与后处理理论. 上海: 上海科技出版社, 1994

18 林群, 严宁宁. 高效有限元的构造与分析. 石家庄: 河北大学出版社, 1996

19 朱起定, 林群. 有限元超收玫理论. 长沙: 湖南科技出版社, 1989

20 Wahlbin L B. Superconvergence in Galerkin Finite Element Methods. Berlin, Heideberg: Spring-Verlag, 1995

\section{附录: 引理 4.2 的证明}

为简便起见, 仅考虑 $L u=-\Delta u$ 的情形, 即

$$
a(u, v)=\int_{\Omega} \nabla u \nabla v,
$$

所有的结果可推广到二阶椭圆方程.

设 $B$ 是半径为 $r$ 的球, $B_{d}$ 是半径为 $r+d$ 的同心球, 熟知若 $u$ 在 $B_{d}$ 内是调和的, 则有

$$
\begin{aligned}
\|\nabla u\|_{L^{2}(B)} & \leqslant C d^{-1}\|u\|_{L^{2}\left(B_{d}\right)}, \\
\left\|u^{h}\right\|_{H^{1}\left(B_{d}\right)} & \leqslant C d^{-1}\left\|u^{h}\right\|_{L^{2}\left(B_{d}\right)} .
\end{aligned}
$$

由文献 [16] 有如下命题: 
命题 1 若 $u^{h} \in S^{h}, B_{d} \subset D \subset \Omega$ 满足

$$
a(u, v)=0, \quad \forall v \in S_{0}^{h}(D),
$$

则

$$
\left\|u^{h}\right\|_{L^{2}(B)}^{2} \leqslant C\left\{h^{2}\left\|\nabla u^{h}\right\|_{L^{2}\left(B_{d}\right)}^{2}+\frac{1}{d^{2}}\left\|u^{h}\right\|_{H^{-1}\left(B_{d}\right)}\right\} .
$$

命题 2 在命题 1 的假设下, 有

$$
\left\|u^{h}\right\|_{1, \infty, B} \leqslant C d^{-2}|\ln h|^{\frac{1}{2}}\left\|u^{h}\right\|_{L^{2}\left(B_{2 d}\right)} .
$$

引理 1 设 $g=\partial_{z}^{i} G_{z}, g^{h}=\partial_{z}^{i} G_{z}^{h}$, 则对任意 $s \leqslant k-1$, 有

$$
\left\|g-g^{h}\right\|_{H^{-s}(\Omega)} \leqslant C h^{s+1-i} .
$$

证明 先设 $g=\partial_{z}^{i} G_{z}^{*}$, 注意到

$$
\left\|g-g^{h}\right\|_{H^{-s}(\Omega)}=\sup _{\varphi \in H^{s}(\Omega)} \frac{\left(g-g^{h}, \varphi\right)}{\|\varphi\|_{H^{s}(\Omega)}} .
$$

设 $\tilde{\varphi} \in H_{0}^{1}(\Omega) \cap H^{s+2}(\Omega)$ 满足

$$
(\nabla \tilde{\varphi}, \nabla v)=(\varphi, v), \quad \forall v \in H_{0}^{1}(\Omega)
$$

显然

$$
\left\|g-g^{h}\right\|_{H^{-s}(\Omega)}=\sup _{\tilde{\varphi} \in H_{0}^{s+2}(\Omega)} \frac{\int_{\Omega} \nabla\left(g-g^{h}\right) \nabla \tilde{\varphi}}{\|\tilde{\varphi}\|_{H^{s+2}(\Omega)}},
$$

并且由引理 3.7 及插值估计有

$$
\begin{aligned}
\int_{\Omega} \nabla\left(g-g^{h}\right) \nabla \tilde{\varphi} & =\int_{\Omega} \nabla\left(g-g^{h}\right) \nabla\left(\tilde{\varphi}-\tilde{\varphi}^{I}\right) \\
& \leqslant\left\|g-g^{h}\right\|_{1}\left\|\tilde{\varphi}-\tilde{\varphi}^{I}\right\|_{1} \\
& \leqslant C h^{-i} C h^{s+1}\|\tilde{\varphi}\|_{H^{s+2}(\Omega)} \\
& =C h^{s+1-i}\|\tilde{\varphi}\|_{H^{s+2}(\Omega)} .
\end{aligned}
$$

最后利用谢锐锋提出的极限过渡的办法 (参见文献 [19, 第五章 $\S 1]$ ) 可以得到, 将 $g=\partial_{z}^{i} G_{z}^{*}$ 换成 $g=\partial_{z}^{i} G_{z}$ 结论依旧成立.

引理 2 设 $g=\partial_{z}^{i} G_{z}, g^{h}=\partial_{z}^{i} G_{z}^{h}$, 存在常数 $C$, 使得当 $k \geqslant 2$ 时有

$$
\left|\nabla\left(g(x)-g^{h}(x)\right)\right| \leqslant C h^{2-i}|\ln h||x-z|^{-3} .
$$

证明 设 $x$ 为 $B$ 的中心, $r=\frac{1}{4}|z-x|, d=\frac{1}{2}|z-x|, \omega$ 为 $B$ 上的截断函数, 令 $u_{1}=\omega g(x), u_{1}^{h}$ 为 $u_{1}$ 的 Galerkin 逼近, $u_{2}^{h}=g^{h}(x)-u_{1}^{h}$, 则

$$
a\left(u_{2}^{h}, v\right)=0, \quad \forall v \in S_{0}^{h}(B),
$$

并且

$$
\left|\nabla\left(g(x)-g^{h}(x)\right)\right| \leqslant\left|u_{1}-u_{1}^{h}\right|_{1, \infty, B}+\left|\nabla u_{2}^{h}(x)\right|=I_{1}+I_{2} .
$$

熟知 ${ }^{[20]}\left|D_{x}^{k} G_{z}(x)\right| \leqslant C|x-z|^{-k}$, 故

$$
I_{1} \leqslant\left\|u_{1}-u_{1}^{h}\right\|_{1, \infty, B_{d}} \leqslant C h^{k}\left|\omega G_{z}^{i}(x)\right|_{k+1, \infty, B_{d}}
$$




$$
\leqslant C h^{k}|x-z|^{-(k+1+i)} \leqslant C h^{2-i}|x-z|^{-3} .
$$

为估计 $I_{2}$, 将 $B$ 做一点小修改, 使得 $\omega$ 在 B 内有支集, 设 $r=\frac{1}{8}|x-z|, d=\frac{1}{8}|x-z|$, 则由命题 1 和 2 有

$$
\begin{aligned}
\left|\nabla u_{2}^{h}(x)\right|^{2} \leqslant & d^{-4}|\ln h|\left\|u_{2}^{h}\right\|_{L^{2}\left(B_{d}\right)}^{2} \\
\leqslant & \leqslant d^{-6}|\ln h|\left(h^{2}\left\|u_{2}^{h}\right\|_{L^{2}\left(B_{d}\right)}^{2}+\left\|u_{2}^{h}\right\|_{H^{-1}\left(B_{d}\right)}^{2}\right) \\
\leqslant & d^{-6}|\ln h|\left\{h^{2}\left\|g-g^{h}\right\|_{L^{2}\left(B_{d}\right)}^{2}+\left\|g-g^{h}\right\|_{H^{-1}\left(B_{d}\right)}^{2}\right. \\
& \left.\quad+h^{2}\left\|u_{1}-u_{1}^{h}\right\|_{L^{2}\left(B_{d}\right)}^{2}+\left\|u_{1}-u_{1}^{h}\right\|_{H^{-1}\left(B_{d}\right)}^{2}\right\} \\
& \leqslant C d^{-6}|\ln h| h^{2}\left\{\left\|g-g^{h}\right\|_{L^{2}\left(B_{d}\right)}^{2}+\left\|u_{1}-u_{1}^{h}\right\|_{L^{2}\left(B_{d}\right)}^{2}\right\} .
\end{aligned}
$$

而由引理 3.9

$$
\begin{aligned}
\left\|g-g^{h}\right\|_{L^{2}\left(B_{d}\right)} & \leqslant\|g\|_{L^{2}\left(B_{d}\right)}+\left\|g^{h}\right\|_{L^{2}\left(B_{d}\right)} \leqslant C h^{1-i}, \\
\left\|u_{1}-u_{1}^{h}\right\|_{L^{2}\left(B_{d}\right)} & \leqslant C h^{k+1}\left|u_{1}\right|_{k+1, B_{d}} \\
& \leqslant C h^{k+1}(r+d)\left|\omega \partial_{z}^{i} G_{z}(x)\right|_{k+1, \infty, B_{d}} \\
& \leqslant C h^{k+1}|x-z \| x-z|^{-(k+1+i)} \leqslant C h^{k+1}|x-z|^{-(k+i)} \leqslant C h^{1-i} .
\end{aligned}
$$

由 (0.7)-(0.11) 式可得 (0.6) 式.

引理 4.2 的证明 设 $x \in e, z \notin e$, 记 $d_{0}=\operatorname{dist}(z, e)$, 则 $d_{0} \geqslant \frac{1}{2}|x-z|$. 应用逆不等式, 逼近理论 及引理 2 有

$$
\begin{aligned}
\left|\partial^{2} \partial_{z}^{i} G_{z}^{h}(x)\right| \leqslant & \left\|\partial^{2} \partial_{z}^{i} G_{z}^{h}(x)-\partial^{2} \partial_{z}^{i} G_{z}^{I}(x)\right\|_{0, \infty, e} \\
& +\left|\partial^{2} \partial_{z}^{i} G_{z}^{I}(x)-\partial^{2} \partial_{z}^{i} G_{z}(x)\right|+\left|\partial^{2} \partial_{z}^{i} G_{z}(x)\right| \\
\leqslant & C h^{-1}\left\|\partial \partial_{z}^{i} G_{z}^{h}(x)-\partial \partial_{z}^{i} G_{z}^{I}(x)\right\|_{0, \infty, e}+C h^{k-2} d_{0}^{-(k+1+i)} \\
& +C|x-z|^{-(2+i)} \\
\leqslant & C h^{-1} C h^{2-i}\left|\ln h\left\|x-\left.z\right|^{-3}+C h^{-1}\right\| \partial \partial_{z}^{i} G_{z}^{I}(x)-\partial \partial_{z}^{i} G_{z}(x) \|_{0, \infty}\right. \\
& +C h^{1-i}|x-z|^{-3} \\
\leqslant & C h^{1-i}|\ln h \| x-z|^{-3} .
\end{aligned}
$$

